\title{
Ogilvie Syndrome in a Refractory Germ Cell Tumor Treated with Vinblastine, Ifosfamide and Cisplatin Regimen
}

\author{
Hrishi Varayathu $^{a} \quad$ Mansi Sandip Shah ${ }^{b}$ Vinu Sarathy ${ }^{b}$ \\ Beulah Elsa Thomas ${ }^{c}$ Vinayak Munirathnam ${ }^{b}$ \\ Shivakumar Swamy Shivalingappa ${ }^{d}$ Radheshyam Naik ${ }^{b}$ \\ aDepartment of Translational Medicine and Therapeutics, Healthcare Global Enterprises \\ Limited, Bangalore, India; ${ }^{b}$ Department of Medical Oncology, Healthcare Global Enterprises \\ Limited, Bangalore, India; 'Department of Clinical Pharmacology, Healthcare Global \\ Enterprises Limited, Bangalore, India; dDepartment of Radiology, Healthcare Global \\ Enterprises Limited, Bangalore, India
}

\author{
Keywords \\ Drug-induced Ogilvie syndrome · Autonomic neuropathy · Peripheral neuropathy · \\ Cumulative toxicity
}

\section{Abstract}

Ogilvie syndrome or intestinal pseudo-obstruction is a clinical syndrome characterized by autonomic imbalance affecting peristalsis of colon leading to obstructive signs and symptoms. The etiologies commonly implicated are drugs affecting the cholinergic system, narcotics, electrolyte imbalance, severe sepsis, cancer, major surgery, and renal and cardiac failure. Ogilvie syndrome secondary to chemotherapy is a very rare phenomenon with very few reports in the literature. Cisplatin-induced neuropathy has been reported to occur when the cumulative dose exceeds $360 \mathrm{mg} / \mathrm{m}^{2}$. It manifests predominantly as peripheral sensory neuropathy with autonomic neuropathy occurring very rarely in a subset of patients. All the reported cases to date who presented with autonomic dysfunction secondary to cisplatin also had peripheral sensory neuropathy. Herein, we report a case of metastatic nonseminomatous germ cell tumor treated with cisplatin based regimen, who presented with severe intestinal pseudo-obstruction when the cumulative dose exceeded $400 \mathrm{mg} / \mathrm{m}^{2}$ without any other manifestation of neuropathy. To our knowledge this is the first such case reported in the literature. 


\section{Background}

Ogilvie syndrome or acute colonic pseudo-obstruction (ACPO) is a rare acquired disorder characterized by autonomic abnormalities affecting peristalsis of the colon. The imbalance in autonomic activity leading to excessive sympathetic stimulation of the colon and suppressed parasympathetic activity has been proposed as the mechanism for this syndrome. Druginduced ACPO is very rare and has been reported with neuroleptic medications, anti-cholinergics, amphetamines, steroids, and narcotics. Cytotoxic chemotherapy such as vincristine, cisplatin and high-dose methotrexate are rarely reported as a cause of Ogilvie syndrome [1-5]. We report a patient with a germ cell tumor who manifested with Ogilvie syndrome after 1 cycle of second-line vinblastine, ifosfamide and cisplatin (VeIP) combination chemotherapy.

\section{Case Presentation}

A 43-year-old male patient diagnosed with non-seminomatous mixed germ cell tumor (yolk sac $80 \%$, teratoma $8 \%$, and embryonal 2\%) categorized as good risk with pulmonary metastasis underwent orchidectomy and 4 cycles of adjuvant bleomycin + etoposide + cisplatin (BEP) regimen. The patient received a cumulative dose of 270 IU of bleomycin, 400 $\mathrm{mg} / \mathrm{m}^{2}$ of cisplatin, and $2 \mathrm{~g} / \mathrm{m}^{2}$ of etoposide after 4 cycles of BEP regimen. The patient developed bleomycin-induced grade 2 pulmonary toxicity after 270 IU; hence, bleomycin was omitted from the fourth cycle of chemotherapy. After 4 cycles of BEP regimen, CT scan showed residual disease in the lung. As the patient was not willing to undergo surgery, he was planned and received a second-line VeIP regimen (vinblastine $9 \mathrm{mg}+$ cisplatin $100 \mathrm{mg} / \mathrm{m}^{2}+$ ifosfamide $6 \mathrm{~g} / \mathrm{m}^{2}$ ). The patient did not have any clinical signs or symptoms of peripheral neuropathy, and audiometry was normal before initiation of VeIP. Three days after completion of first cycle of VeIP regimen, the patient presented with complaints of severe abdomen pain and constipation. X-ray erect abdomen showed grossly distended large bowel loops with fecal matter and normal bowel gas pattern (shown in Fig. 1). Blood investigations showed normal serum electrolytes and renal and hepatic function. Serum amylase and lipase levels were also within normal limits. There were no signs of active infection like fever or diarrhea, and blood counts were within normal limits. The patient was not on any medication known to cause paralytic ileus like anti-cholinergics, opioids or any anti-motility drugs. On physical examination, the patient had diffuse tenders distension of the abdomen with decreased bowel sounds. There was no evidence of free fluid on percussion. The patient was afebrile with a slightly elevated blood pressure of 154/90 $\mathrm{mm} \mathrm{Hg}$ and had tachycardia with a heart rate of $122 /$ min. All other vital signs were within normal limits. As the patient's abdominal pain was very severe (visual analogue scale 9/10) and did not respond to analgesics, a CT scan abdomen was done which showed extensive fecal loading in the caecum and ascending and transverse colon without any evidence of obstruction (Fig. 2). Per rectal examination was done which showed an empty rectum. The patient was treated with laxatives, enema and fentanyl infusion for severe pain. With the above supportive measures, the patient gradually recovered and was relieved of his constipation and pain 1 week after the first cycle of VeIP regimen.

Outcome and Follow-Up

Chemotherapy with VeIP was discontinued, the and patient is planned for radiotherapy to residual lung lesions.

\section{Karger'k}




\section{Case Reports in Oncology}
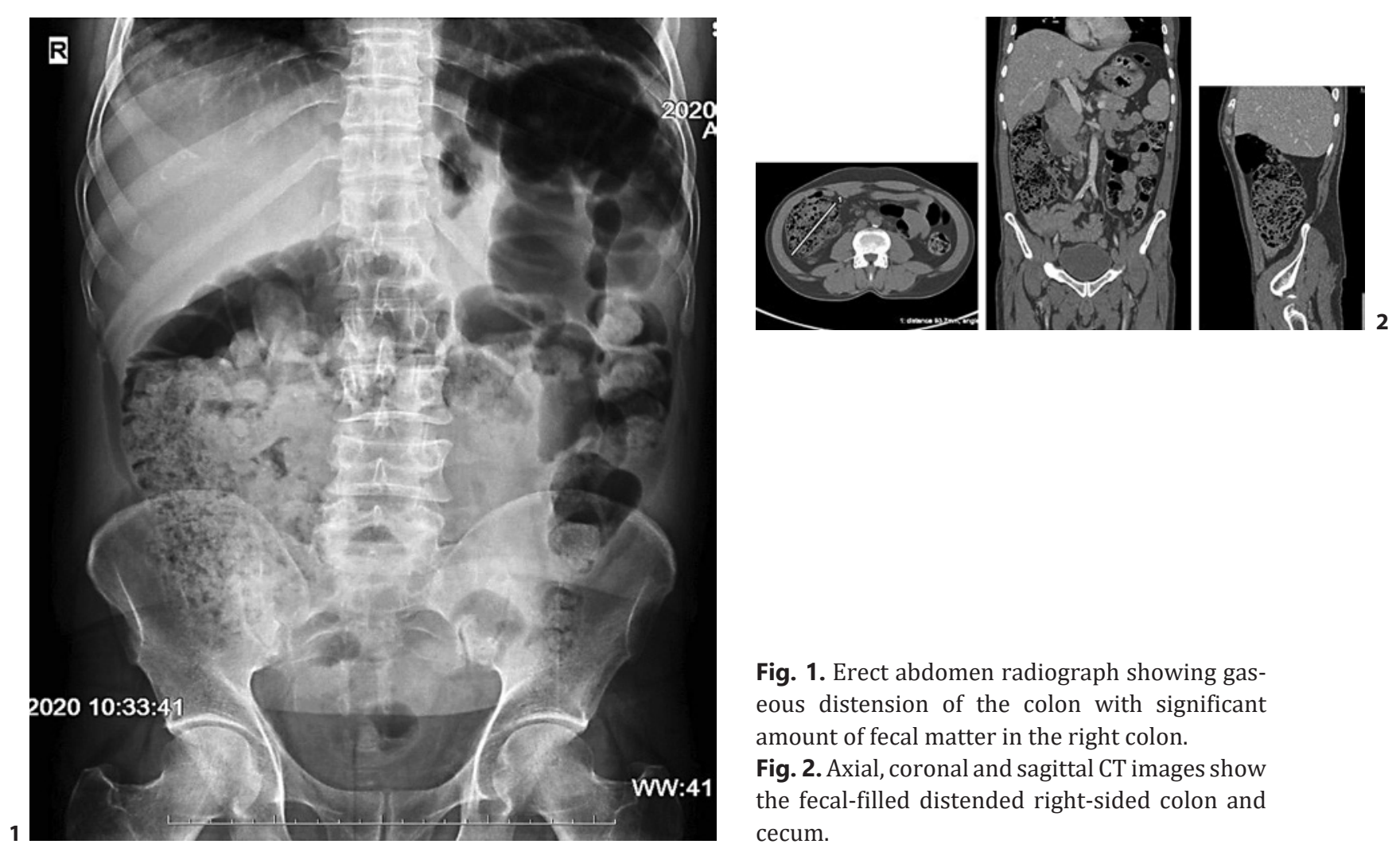

Fig. 1. Erect abdomen radiograph showing gaseous distension of the colon with significant amount of fecal matter in the right colon.

Fig. 2. Axial, coronal and sagittal CT images show the fecal-filled distended right-sided colon and cecum.

\section{Discussion}

Ogilvie syndrome or ACPO is a disorder of gastrointestinal motility due to autonomic imbalance or autonomic nerve dysfunction which is characterized by dilation of colon without any mechanical obstruction. The exact cause and underlying mechanism of Ogilvie syndrome is not fully understood. The disorder is most commonly associated with serious medical illness or surgical procedures. Medical conditions such as nonoperative trauma, infections, electrolyte imbalance, renal insufficiency, heart diseases, respiratory failure, cancer and surgeries including abdominal, orthopedic (especially total hip replacement), neurologic, urologic and cardiac surgeries have been reported to be associated with Ogilvie syndrome [3]. However, cytotoxic drug-induced ACPO is a very rare complication and is usually accompanied by concomitant peripheral neuropathies. Peripheral neuropathy is a common doselimiting toxicity with various cytotoxic drugs including cisplatin and vinca alkaloids. The mechanism of chemotherapy-induced peripheral neuropathy is multifactorial and involves microtubule disruption, oxidative stress, mitochondrial damage, altered ion channel activity, myelin sheath damage, DNA damage, immunological processes and neuro-inflammation [6]. Peripheral neuropathies can impair sensory, motor and autonomic function either singly or in combination. Neuropathies associated with chemotherapy are dose dependent. As our patient received only one dose of vinblastine, which is known to have very low neurotoxic potential, we hypothesized that our case of ACPO was mainly due to cisplatin as patient had already received a cumulative dose of $400 \mathrm{mg} / \mathrm{m}^{2}$ prior to second line therapy with VeIP. Krarup-Hansen et al. [7]reported that neuropathy occurs with cumulative dose $>350 \mathrm{mg} / \mathrm{m}^{2}$ of cisplatin which is greatly increased beyond cumulative doses of $500-600 \mathrm{mg} / \mathrm{m}^{2}$. Kurita et al. [8] reported that cisplatin-induced autonomic nervous dysfunction manifests after a 
patient has received cumulative dose sufficient enough to produce severe sensory neuropathy. Our patient developed ACPO after a cumulative cisplatin dose of $500 \mathrm{mg} / \mathrm{m}^{2}$ without any evidence of sensory neuropathy. Abd Rahim and Fuang Ho et al. [2] reported a case of paralytic ileus 3 days after completion of cisplatin, which was similar to our case. Cases of Ogilvie syndrome reported in association with drugs have been mostly treated with para-sympathomimetic agents like neostigmine or spontaneously relieved as the drug effect wears off. In our case, patient gradually recovered 7 days after last dose of cisplatin. The patient most probably recovered as the effects of cisplatin gradually wore off.

In a nutshell, Ogilvie syndrome associated with cisplatin is extremely rare, and caution should be exercised especially when cumulative cisplatin dose exceeds $400 \mathrm{mg} / \mathrm{m}^{2}$. Concomitant use of other potentially neurotoxic drugs like vinca alkaloids may further accentuate this cytotoxicity.

\section{Acknowledgement}

We acknowledge all the team members of Department of Medical Oncology Healthcare Global enterprises limited for sharing their knowledge and valuable inputs.

\section{Statement of Ethics}

The research was conducted ethically in accordance with the World Medical Association Declaration of Helsinki. The subject has given his written informed consent to publish his case.

\section{Conflict of Interest Statement}

The authors have no conflicts of interest to declare.

\section{Funding Sources}

No funding has been received for the study.

\section{Author Contributions}

Hrishi Varayathu: manuscript writer, design of the study, observation. Mansi Sandip Shah: treating oncologist. Vinu Sarathy: treating oncologist, observation. Beulah Elsa Thomas: manuscript writing, references, pharmacology inputs. Vinayak Munirathnam: treating oncologist. Shivakumar Swamy Shivalingappa: radiologist. Radheshyam Naik: consultant, proof reading, final approval.

\section{Karger'}




\section{References}

1 Carraro F, Rivetti E, Romano E, Fagioli F. Two cases of paralitic ileus in onco-hematologic patients. Pediatr Rep. 2012;4(1):e3.

2 Case report: An unusual case of cisplatin induced paralytic ileus | Rahim | Advances in Modern Oncology Research [Internet]. [cited 2020 May 31]. Available from: http://www.advmodoncolres.sg/index.php/amor/ article/view/159/162

3 Lee GE, Lim G-Y, Lee J-W, Cho B. Acute colonic pseudo-obstruction complicating chemotherapy in paediatric oncohaematological patients: clinical and imaging features.

4 Xie H, Peereboom DM. Ogilvie's syndrome during chemotherapy with high-dose methotrexate for primary CNS lymphoma. J Clin Oncol. 2012 Jul 20 [cited 2020 May 31];30(21):e192.

5 Pessôa FMC, Bittencourt LK, de Melo ASA. Síndrome de Ogilvie após uso de vincristina: Achados tomográficos. Radiologia Brasileira. Colegio Brasileiro de Radiologia. 2017; Vol. 50:273-4.

6 Zajaczkowska R, Kocot-Kepska M, Leppert W, Wrzosek A, Mika J, Wordliczek J. Molecular Sciences Mechanisms of Chemotherapy-Induced Peripheral Neuropathy; 2019 [cited 2020 May 31] www.mdpi.com/journal/ ijms.

7 Krarup-Hansen A, Helweg-Larsen S, Schmalbruch H, Roth M, Krarup C. Neuronal involvement in cisplatin neuropathy: prospective clinical and neurophysiological studies | Brain | Oxford Academic [Internet]. [cited 2020 May 31]. https://academic.oup.com/brain/article/130/4/1076/275387

8 Kurita A, Mochio S, Hasunuma T, Oka H, Isogai Y. Cisplatin-induced autonomic nervous dysfunction. Journal of the Autonomic Nervous System. 1995 Jan 3;50(3):372. 